\title{
ON THE IWASAWA INVARIANTS OF IMAGINARY ABELIAN FIELDS
}

\author{
TAUNO METSÄNKYLÄ
}

\section{Introduction}

Let $E$ be an absolutely abelian number field and let $p$ be a prime. A Galois extension of $E$ is called a $Z_{p}$-extension if its Galois group is isomorphic to the additive group of $Z_{p}$, the ring of $p$-adic integers.

Fix a natural number $m$ not divisible by $p$. For $n \geqq 0$, denote by $F_{n}$ the cyclotomic field of $m q p^{n}$ th roots of unity, where $q=p$ if $p>2$ and $q=4$ if $p=2$. If $E=F_{0}$, then the union $F_{\infty}$ of all the fields $F_{n}$ is a $Z_{p}$-extension of $E$. More generally, if $E$ is a subfield of $F_{0}$ with conductor $m$ or $m q$, then there exists a unique $\boldsymbol{Z}_{p}$-extension $E_{\infty}$ of $E$, called the basic $Z_{p}$-extension, which is contained in $F_{\infty}$.

Denote by $\lambda=\lambda(E)$ and $\mu=\mu(E)$ the Iwasawa invariants of $E$, i.e. the Iwasawa invariants of the basic $Z_{p}$-extension $E_{\infty} / E$. It is well known that $\lambda$ and $\mu$ are non-negative integers having the following connection with the class numbers $h_{n}$ of the intermediate fields $E_{n}$ of $E$ and $E_{\infty}$ : if $\left[E_{n}: E\right]=p^{n}$ and if the highest power of $p$ dividing $h_{n}$ is $p^{e(n)}$, then, for all sufficiently large $n, e(n)$ is of the form $\lambda n+\mu p^{n}+v$, where $v$ is also an integer independent of $n$. Iwasawa [9] has conjectured that $\mu=0$ for every $E$ and $p$; the conjecture has been proved by B. Ferrero in the cases $p=2$ and $p=3$ (as yet unpublished).

In what follows we shall assume that $E$ is imaginary, and put $\lambda=\lambda^{+}+\lambda^{-}, \mu=\mu^{+}+\mu^{-}$, where $\lambda^{+}$and $\mu^{+}$are the Iwasawa invariants of the maximal real subfield of $E$. Thus, if $p^{a(n)}$ denotes the highest power of $p$ dividing the first factor of the class number of $E_{n}$, we have $a(n)=\lambda^{-} n+\mu^{-} p^{n}+v^{-}\left(v^{-}\right.$an integer $)$for all large $n$. While the normal approach to $\lambda$ and $\mu$ is via the general theory of $Z_{p}$ extensions (see [6], [10]), there is also another way of introducing $\lambda^{-}$and $\mu^{-}$, namely the use of Iwasawa's theory of $p$-adic $L$-functions (see [7]). This method has been applied by Iwasawa [8] in case $E=F_{0}$. In the 
present note we shall first apply the same method to the case of a general $E$ and an arbitrary odd prime $p$, and show the existence of the invariants $\lambda^{-}$and $\mu^{-}$as sums of certain components which arise naturally from considering the characters of $E$. Furthermore, using results from [11] we shall obtain immediately a criterion for the vanishing of $\mu^{-}$. This criterion, together with some facts proved essentially in [11], will then be applied to give a relationship between the invariants $\lambda^{-}$and $\mu^{-}$of two abelian fields of a certain type.

\section{Characters and $p$-adic $L$-functions}

Throughout the following, let $p$ be a fixed odd prime. As usual, let $Z, Q, Z_{p}$, and $Q_{p}$ stand for the ring of rational integers, the field of rational numbers, the ring of $p$-adic integers, and the field of $p$-adic numbers, respectively. Denote by $|\cdot|$ the $p$-adic valuation on a fixed algebraic closure $\Omega_{p}$ of $Q_{p}$.

Let $\chi$ be a Dirichlet character. We shall always assume that $\chi$ is primitive, and denote its conductor by $f_{\chi}$. Let $U(f)$ denote the group of all characters $\chi$ with $f_{\chi} \mid f$.

Suppose that $m$ is a natural number prime to $p$. For each $n \geqq 0$, denote by $G_{n}$ the multiplicative residue class $\operatorname{group} \bmod m p^{n+1}$, consisting of all elements $\sigma_{n}(a)=a+m p^{n+1} \boldsymbol{Z}$, where $(a, m p)=1$. It is known that $G_{n}=\Delta_{n} \times \Gamma_{n}$ (direct product), where

$$
\begin{gathered}
\Delta_{n}=\left\{\sigma_{n}(a) \mid a^{p-1} \equiv 1\left(\bmod p^{n+1}\right)\right\}, \\
\Gamma_{n}=\left\{\sigma_{n}(a) \mid a \equiv 1(\bmod m p)\right\}
\end{gathered}
$$

(see e.g. [5], pp. $78-81,[8]$, p. 67). From this it follows that

$$
U\left(m p^{n+1}\right)=U(m p) \times T_{n},
$$

where $T_{n}$ is the group of all characters $\pi$ satisfying the conditions $f_{\pi} \mid p^{n+1}$ and $\pi(a)=1$ whenever $\sigma_{n}(a) \in \Delta_{n}$. Usually the elements of $U(m p)$ and $T_{n}$ are called characters of first and second kind, respectively. Note that $T_{n}$ is a cyclic group of order $p^{n}$.

The unit group of $\boldsymbol{Z}_{p}$ can be written in the form $\boldsymbol{V} \times \boldsymbol{D}$, where $\boldsymbol{V}$ is the group of all $(p-1)$ st roots of unity and $\boldsymbol{D}$ the group of principal units. For any $p$-adic unit $a$, let $\omega(a)$ denote the projection of $a$ on $\boldsymbol{V}$ under this decomposition. Then $\omega$ can be regarded, in an obvious manner, as a character with order $p-1$ and conductor $p$. In particular, $\omega \in U(m p)$.

Now let $L_{p}(s ; \chi)$ be the $p$-adic $L$-function for an even character 
$\chi \in U\left(m p^{n+1}\right)$. For our purposes it suffices to consider the value of $L_{p}(s ; \chi)$ at $s=0$; a well-known formula ([8], p. 30) asserts that

$$
L_{p}(0 ; \chi)=-\left(1-\left(\chi \omega^{-1}\right)(p)\right) B_{1}\left(\chi \omega^{-1}\right),
$$

where $B_{1}(\chi)$ denotes the first generalized Bernoulli number belonging to the character $\chi$. Put $\chi=\theta \pi$ with $\theta \in U(m p)$ and $\pi \in T_{n}$; then $f_{\theta}=m_{0}$ or $m_{0} p$ with $m_{0} \mid m$. Let $K$ be a finite extension of $Q_{p}$ in $\Omega_{p}$, containing the numbers $\theta(a)$ for all $a \in Z$, and let $\mathfrak{D}$ be the ring of local integers in $K$. It follows from Iwasawa's theory of $p$-adic $L$-functions that

$$
L_{p}(0 ; \chi)=2 f\left(\pi\left(1+m_{0} p\right)^{-1}-1 ; \theta\right)
$$

([8], p. 87), where $f(x ; \theta)$ is a certain power series with coefficients in $\mathfrak{v}$, if $\theta \neq \chi_{0}$ (i.e., $\theta$ is non-principal), and $f\left(x ; \chi_{0}\right)$ is a quotient of two such power series.

\section{The invariants $\lambda^{-}$and $\mu^{-}$of imaginary abelian fields}

Let $E / Q$ be a finite imaginary abelian extension. When investigating the invariants $\lambda^{-}$and $\mu^{-}$of the basic $Z_{p}$-extension $E_{\infty} / E$ we may assume without loss of generality that the conductor of $E$ is of the form $m$ or $m p$.

Let $F_{0}, F_{1}, \ldots$ denote the cyclotomic fields defined in the introduction. We shall identify $\operatorname{Gal}\left(F_{n} / Q\right)$, the Galois group of $F_{n} / Q$, with the group $G_{n}$ in the usual manner. Then

$$
\operatorname{Gal}\left(F_{n} / F_{0}\right)=\Gamma_{n}, \quad \operatorname{Gal}\left(F_{0} / Q\right)=G_{0}=\Delta_{0} .
$$

Moreover, the character group $\mathrm{Ch}\left(F_{n}\right)$ belonging to the extension $F_{n} / Q$ is $U\left(m p^{n+1}\right)$.

Put $Y=\mathrm{Ch}(E)$ and note that $Y$ is a subgroup of $U(m p)$. Let

$$
E=E_{0} \subset E_{1} \subset \ldots \subset E_{n} \subset \ldots
$$

be the infinite tower of fields determining the $Z_{p}$-extension $E_{\infty} / E$. Then we have $\operatorname{Gal}\left(E_{n} / E\right) \simeq \Gamma_{n}$ and

$$
F_{n}=F_{0} E_{n}, \quad E=F_{0} \cap E_{n}
$$

for every $n \geqq 0$. Hence $\mathrm{Gal}\left(F_{n} / E_{n}\right)$ is a subgroup of $\Delta_{n}$ and it follows easily that

$$
\operatorname{Ch}\left(E_{n}\right)=Y \times T_{n} .
$$

Now let

$$
X=X(E)=\left\{\theta \omega \mid \theta \in Y^{-}, \theta \neq \omega^{-1}\right\},
$$


where by $Y^{-}$is meant the subset of $Y$ consisting of all odd characters. Note that the set $X$ is empty if and only if $E$ is the cyclotomic field of 3rd roots of unity. Let $h_{n}$ and $h_{n}^{+}$denote the class numbers of $E_{n}$ and its maximal real subfield, respectively. We shall prove the following lemma on $h_{n}^{-}=h_{n} / h_{n}^{+}$.

$\mathrm{L}$ e $\mathrm{m}$ a 1 . Put

then

$$
A(x)=\prod_{\theta \in X} f(x ; \theta)
$$

$$
\left|h_{n}^{-}\right| h_{0}^{-}|=| \prod_{\zeta \in W_{n}} A(\zeta-1) \mid \quad(n \geqq 1),
$$

where $W_{n}$ denotes the set of all $p^{n}$ th roots of unity except 1.

Proof. We start from the formula ([4], p. 12)

$$
h_{n}^{-}=Q_{n} w_{n} \prod_{\chi}\left(-\frac{1}{2 f_{\chi}} \sum_{a=1}^{f_{\chi}} a \chi(a)\right) \quad(n \geqq 0),
$$

where $Q_{n}=1$ or $2, w_{n}$ is the number of roots of unity in $E_{n}$, and the product is extended over all odd characters $\chi$ in $Y \times T_{n}$. Let us fix a generator $\pi_{n}$ of $T_{n}$. Noting that

$$
f_{\chi}^{-1} \sum_{a=1}^{f_{\chi}} a \chi(a)=B_{1}(\chi)
$$

(see e.g. [8], p. 14) we then obtain

$$
\left|h_{n}^{-} / h_{0}^{-}\right|=\left|\left(w_{n} / w_{0}\right) \underset{\theta \in Y^{-}}{\prod_{k=1}^{p^{n}-1}}\left(-\frac{1}{2} B_{1}\left(\theta \pi_{n}^{k}\right)\right)\right| \quad(n \geqq 1) .
$$

Consider a character $\chi=\theta \omega \pi_{n}^{k}$ with $\theta \in Y^{-}, 1 \leqq k \leqq p^{n}-1$. We have $\chi(-1)=1,\left(\chi \omega^{-1}\right)(p)=0$ and $f_{\theta \omega}=m_{0}$ or $m_{0} p$, where $m_{0} \mid m$. Hence, by combining (1) and (2) we find that

$$
-B_{1}\left(\theta \pi_{n}^{k}\right)=2 f\left(\zeta_{\chi}-1 ; \theta \omega\right) \quad\left(\zeta_{\chi}=\pi_{n}\left(1+m_{0} p\right)^{-k}\right) .
$$

It is easy to see that $\zeta_{\chi}$ ranges over $W_{n}$ as $k$ runs through $1, \ldots, p^{n}-1$. Thus (3) becomes

$$
\left|h_{n}^{-} / h_{0}^{-}\right|=\left|\left(w_{n} / w_{0}\right) \prod_{\theta \in Y^{-}} \prod_{\zeta \in W_{n}} f(\zeta-1 ; \theta \omega)\right| \quad(n \geqq 1) .
$$

Now we have to distinguish between two cases, according to whether $E$ contains the $p$ th roots of unity or not.

Assume first that $W_{1} \subset E$. Then $\omega \in Y^{-}$and the right hand side of (4) contains the factor

$$
\left|\prod_{\zeta \in W_{n}} f\left(\zeta-1 ; \chi_{0}\right)\right|=\left|p^{n}\right|^{-1}
$$


([8], p. 92). On the other hand, $w_{n}=w_{0} p^{n}$, so that the assertion of the lemma follows.

If $W_{1}$ is not contained in $E$, we have $\omega \notin Y^{-}$and $w_{n}=w_{0}$. Thus our assertion is immediate from (4).

Remark 1. If $|A(\zeta-1)|<1$ for some $\zeta \in W_{n}$, then $|A(0)|<1$ and so $|A(\zeta-1)|<1$ for every $\zeta \in W_{n}$. Consequently, it follows from Lemma 1 that

(i) $p$ divides $h_{n}^{-} / h_{0}^{-} \quad(n \geqq 1)$ if and only if $p$ divides $h_{1}^{-} / h_{0}^{-}$;

(ii) if $p$ divides $h_{n}^{-} / h_{0}^{-} \quad(n \geqq 1)$, then $p^{n}$ divides $h_{n}^{-} / h_{0}^{-}$.

In particular, if $m=1$ (i.e., $E$ is a subfield of the cyclotomic field of $p$ th roots of unity), then an obvious modification of the proof of Lemma 1 leads to the formula

$$
\left|h_{n}^{-}\right|=\left|\prod_{\zeta \in W_{n} \cup\{1\}} A(\zeta-1)\right| \quad(n \geqq 0),
$$

so that we obtain the following simpler results:

(i') $p \mid h_{n}^{-}$if and only if $p \mid h_{0}^{-}$;

(ii') if $p \mid h_{n}^{-}$, then $p^{n+1} \mid h_{n}^{-}$.

Another proof for (i') and (ii') has been given by Adachi [1].

Now suppose that the field $K$ associated with the power series $f(x ; \theta)$ is the extension of $Q_{p}$ generated by all numbers $\theta(a)$, where $\theta \in X(E)$ and $a \in Z$. Let $e$ denote the ramification index of $K / Q_{p}$, let $\pi$ be a fixed prime element of the ring $\mathfrak{D}$, and let $\mathfrak{p}=\pi \mathfrak{v}$.

L e m m 2. For $\theta \in X(E)$, define non-negative integers $\lambda_{\theta}=\lambda_{\theta}(E)$ and $\mu_{\theta}=\mu_{\theta}(E)$ by

Then the numbers

$$
\begin{gathered}
f(x ; \theta)=\pi^{\mu_{\theta}} \sum_{k=0}^{\infty} \alpha_{k} x^{k} \quad\left(\alpha_{k} \in \mathfrak{v}\right), \\
\alpha_{k} \equiv 0(\bmod \mathfrak{p}) \text { for } 0 \leqq k<\lambda_{\theta}, \\
\alpha_{k} \neq \equiv 0(\bmod \mathfrak{p}) \text { for } k=\lambda_{\theta} .
\end{gathered}
$$

$$
\lambda^{-}=\sum_{\theta \in X} \lambda_{\theta}, \quad \mu^{-}=e^{-1} \sum_{\theta \in X} \mu_{\theta}
$$

are the invariants $\lambda^{-}(E), \mu^{-}(E)$ of the extension $E_{\infty} / E$.

Proof. We have

where

$$
A(x)=\prod_{\theta \in X} f(x ; \theta)=\pi^{e \mu^{-}} B(x),
$$

$$
B(x)=\sum_{k=0}^{\infty} \beta_{k} x^{k} \quad\left(\beta_{k} \in \mathfrak{v}\right)
$$


with $\beta_{k} \equiv 0(\bmod \mathfrak{p})$ for $0 \leqq k<\lambda^{-}$and $\beta_{k} \neq \equiv 0(\bmod \mathfrak{p})$ for $k=\lambda^{-}$. Let $t$ be the least non-negative integer such that $e \lambda^{-}<(p-1) p^{t}$. Assuming that $n>t$ and $\zeta \in W_{n}-W_{n-1}$ we then find that $|\pi|<|\zeta-1|^{\lambda-}$ and so, furthermore,

$$
|A(\zeta-1)|=\left|\pi^{e \mu^{-}} B(\zeta-1)\right|=\left|p^{\mu^{-}}\right||\zeta-1|^{\lambda^{-}} .
$$

This together with Lemma 1 yields

$$
\left|h_{n}^{-}\right|=\left|p^{a(n)}\right|, \quad a(n)=\lambda^{-} n+\mu^{-} p^{n}+v^{-} \quad(n>t),
$$

where $v^{-}$is an integer independent of $n$.

Remark 2. Suppose that $E \subset E^{\prime}$, where $E^{\prime}$ is abelian with conductor $m^{\prime}$ or $m^{\prime} p, m^{\prime}$ being prime to $p$. Then $X(E) \subset X\left(E^{\prime}\right)$. Therefore, if $\theta \in X(E)$ then $\lambda_{\theta}(E)=\lambda_{\theta}\left(E^{\prime}\right)$ and $e^{\prime} \mu_{\theta}(E)=e \mu_{\theta}\left(E^{\prime}\right)$, where $e^{\prime}$ is the ramification index of the extension $K^{\prime} / Q_{p}$ determined by $X\left(E^{\prime}\right)$.

It should be noted that the numbers $\lambda_{\theta}\left(F_{0}\right), \mu_{\theta}\left(F_{0}\right)$ have been introduced and investigated by the author in [11]. (Cf. [11], Remark (ii) of Section 4.)

\section{On the vanishing of $\lambda^{-}$and $\mu^{-}$}

As an immediate consequence of Remark 1 we may state that the condition

$$
\lambda^{-}=\mu^{-}=0
$$

is equivalent to $\left(p, h_{1}^{-} / h_{0}^{-}\right)=1$, and if $m=1,(5)$ is equivalent to $\left(p, h_{0}^{-}\right)=1$. The latter statement is also implied by the following stronger result, proved essentially in [11] (Lemma 2): for $\theta=\omega^{u} \in X(E)$, $\lambda_{\theta}(E)=\mu_{\theta}(E)=0$ if and only if the $u$ th Bernoulli number $B_{u}$ is prime to $p$. Indeed, if $m=1$ then $\left(p, h_{0}^{-}\right)=1$ is equivalent to the fact that the numbers $B_{u}$ are prime to $p$ whenever $\omega^{u} \in X(E)$ (see [2], [1]).

We shall now give a general criterion for the vanishing of $\mu_{\theta}(E)$. For $n \geqq 0$, let $\gamma_{n}(a)$ be the projection of $\sigma_{n}(a)$ on $\Gamma_{n}$ under the direct decomposition $G_{n}=\Delta_{n} \times \Gamma_{n}$, so that $\gamma_{n}(a)$ runs through the elements of $\Gamma_{n}$, say $g_{n k}\left(k=0, \ldots, p^{n}-1\right)$, as $\sigma_{n}(a)$ runs through $G_{n}$. For $\theta \in X(E)$, put

$$
\xi_{n}=-\left(2 m p^{n+1}\right)^{-1} \sum_{\substack{a=1 \\(a, m p)=1}}^{m p^{n+1}} a \theta(a) \omega^{-1}(a) \gamma_{n}(a)^{-1}=\sum_{k=0}^{p^{n}-1} S_{n k} g_{n k} .
$$

We know that $\xi_{n}$ belongs to the group algebra $\mathfrak{D}\left[\Gamma_{n}\right]$, i.e. the numbers $S_{n k}=S_{n k}(\theta ; E)$ belong to $\mathfrak{D}$, and that $\xi_{n+1} \mapsto \xi_{n}$ under the morphism $\mathfrak{o}\left[\Gamma_{n+1}\right] \rightarrow \mathfrak{D}\left[\Gamma_{n}\right]$, induced by $\sigma_{n+1}(a) \mapsto \sigma_{n}(a)$ ([8], pp. 72-76). Let $R$ 
denote the inverse limit of the $\mathfrak{v}\left[\Gamma_{n}\right]$ with respect to these morphisms. Then $\xi=\lim \xi_{n}$ is a well-defined element of $R$ and closely connected with the power series $f(x ; \theta) \in \mathfrak{v}[[x]]$. Indeed, $\xi$ is the image of $f(x ; \theta)$ under the unique isomorphism $\tau: \mathfrak{D}[[x]] \rightarrow R$, determined by the condition $\tau(1+x)=\lim \gamma_{n}(1+m p)$. This enables us to formulate the following lemma (see [11], Lemma 5).

$\mathrm{L}$ e $\mathrm{m} \mathrm{m}$ a 3. A necessary and sufficient condition for $\mu_{\theta}(E)>0$ is that

$$
S_{n k}(\theta ; E) \equiv 0(\bmod \mathfrak{p})
$$

for all $n \geqq 0$ and all $k \in I_{n}=\left\{0, \ldots, p^{n}-1\right\}$.

From this it is seen that $\mu^{-}(E)>0$ if and only if there is at least one $\theta \in X(E)$ such that the infinite system of congruences (6) is satisfied.

Remark 3. If $\theta \in X(E)$, then $\lambda_{\theta}(E)=\mu_{\theta}(E)=0$ is equivalent to the condition $S_{00}(\theta ; E) \not \equiv 0(\bmod \mathfrak{p})$. This is proved in [11] under the as sumption that the conductors of $\theta$ and $\theta \omega^{-1}$ are equal to $m p$. However, this restriction is unnecessary, since an inspection of the above isomorphism $\tau$ shows that, in any case,

$$
f(0 ; \theta)=S_{00}(\theta ; E) .
$$

Remark 4. A sufficient condition for $\mu_{\theta}(E)>0$ is that

$$
S_{n k}(\theta ; E) \equiv S_{n 0}(\theta ; E)(\bmod \mathfrak{p})
$$

for all $n \geqq 1$ and all $k \in I_{n}$, as can be verified in the following way. By considering the morphism $\mathfrak{D}\left[\Gamma_{n+1}\right] \rightarrow \mathfrak{D}\left[\Gamma_{n}\right]$ mentioned above one finds that

$$
S_{n k}=\sum_{h} S_{n+1, h}
$$

the sum being extended over those $h \in I_{n+1}$ for which $g_{n+1, h} \mapsto g_{n k}$. Obviously, the number of such $h$ is $p$, so that

$$
S_{n k} \equiv \sum_{h} S_{n+1,0} \equiv p S_{n+1,0} \equiv 0(\bmod \mathfrak{p}) \quad\left(n \geqq 0, k \in I_{n}\right) .
$$

For the rest of this section we assume that $m=1$ and $p>3$. Denote by $P$ the cyclotomic field of $p$ th roots of unity; then

$$
X(P)=\left\{\omega^{u} \mid u=2,4, \ldots, p-3\right\}
$$

and $E$ is a subfield of $P$.

We shall introduce some further notation. Let $s=(p-1) / 2$, let $r$ be a primitive root $\bmod p^{n+1}$ for all $n \geqq 0$, and let $r_{n}(i)$ be the least positive residue of $r^{i}\left(\bmod p^{n+1}\right)$. Denote by $\alpha$ the primitive $(p-1)$ st root of unity satisfying

$$
\omega(a)=\alpha^{i} \quad \text { for } a \equiv r^{i}(\bmod p) .
$$


For rational integers $h$ and $u$, put

$$
R_{n}(h, u)=\sum_{i=0}^{p-2} r_{n}\left(i p^{n}+h\right) \alpha^{i(u-1)} \quad(n \geqq 0) .
$$

Then we have the following supplement to Lemma 3.

$\mathrm{L} \mathrm{e} \mathrm{m} \mathrm{ma} \mathrm{4.} \mathrm{If} E \subset P$ and $\omega^{u} \in X(E)$, then

$$
-2 p^{n+1} S_{n k}\left(\omega^{u} ; E\right)=R_{n}(k, u) \alpha^{k(u-1)} \quad\left(n \geqq 0, k \in I_{n}\right),
$$

provided the elements $g_{n k}$ of $\Gamma_{n}$ are suitably ordered.

For the proof, see [11], proof of Lemma 8.

It will be useful to notice that $R_{n}(h, u)$ satisfies the conditions

(8) $R_{n}(h, u) \alpha^{j(u-1)}=R_{n}(g, u) \quad$ for $h \equiv j p^{n}+g\left(\bmod (p-1) p^{n}\right)$.

\section{An example : the invariants of a quadratic field}

Let $E=Q\left((-3)^{1 / 2}\right)$ and $p>3$. Then $X(E)$ contains only one element, namely $\theta \omega$, where $\theta(a)=(a / 3)$ (the Legendre symbol). Hence, in this case $\mathfrak{o}=Z_{p}$. Moreover,

$$
\lambda^{+}(E)=\lambda(Q)=0, \quad \mu^{+}(\mathrm{E})=\mu(Q)=0
$$

([6], p. 225) and so $\lambda(E)=\lambda^{-}(E)=\lambda_{\theta \omega}(E), \mu(E)=\mu^{-}(E)=\mu_{\theta \omega}(E)$. We shall first employ Lemma 3 to prove that $\mu(E)=0$.

Now

$$
S_{n k}=-\left(6 p^{n+1}\right)^{-1} \sum_{a} a(a / 3),
$$

where the summation is extended over the values of $a$ satisfying the conditions $1 \leqq a<3 p^{n+1}, \quad(a, 3 p)=1, \quad \gamma_{n}(a)^{-1}=g_{n k}$. Let $c_{n 1}(i)$ and $c_{n 2}(i)$ be positive integers less than $3 p^{n+1}$ such that

$$
\begin{gathered}
c_{n 1}(i) \equiv c_{n 2}(i) \equiv r^{i}\left(\bmod p^{n+1}\right), \\
c_{n 1}(i) \equiv 1, c_{n 2}(i) \equiv-1(\bmod 3) .
\end{gathered}
$$

Then $\Delta_{n}$ can be written in the form

$$
\Delta_{n}=\left\{\sigma_{n}(a) \mid a=c_{n 1}\left(i p^{n}\right) \text { or } a=c_{n 2}\left(i p^{n}\right), i=0, \ldots, p-2\right\} .
$$

After a suitable rearrangement of the elements $g_{n k}$ of $\Gamma_{n}$ we therefore get

$$
S_{n k}=-\left(6 p^{n+1}\right)^{-1} \sum_{i=0}^{p-2}\left[c_{n 1}\left(i p^{n}+k\right)-c_{n 2}\left(i p^{n}+k\right)\right] \text {. }
$$


Furthermore, $c_{n 1}\left(i p^{n}+k\right)-c_{n 2}\left(i p^{n}+k\right)= \pm b_{i} p^{n+1}$, where each $b_{i}$ is equal to 1 or 2 and, as is easy to see, $b_{i}=b_{i+s}$. Hence

$$
S_{n k}=-\frac{1}{3} \sum_{i=0}^{s-1}\left( \pm b_{i}\right), \quad\left|\sum_{i=0}^{s-1}\left( \pm b_{i}\right)\right|<p .
$$

Now, if $\mu>0$ then Lemma 3 shows that all the numbers $S_{n k}$ vanish. But this would imply that $f(x ; \theta \omega)=0$, which is impossible by Lemma 1 . Consequently, $\mu=0$.

We are also able to determine those primes $p$ for which $\lambda=0$. In fact, using (2) and (1) we may calculate $f(0 ; \theta \omega)$ as follows:

$$
f(0 ; \theta \omega)=\frac{1}{2} L_{p}(0 ; \theta \omega)=-\frac{1}{2}(1-(p / 3)) B_{1}(\theta)=(1-(p / 3)) / 6 .
$$

From this we infer that $\lambda=0$ if and only if $p \equiv 2(\bmod 3)$.

We note that the Iwasawa invariants of imaginary quadratic fields have been examined by Gold in several papers (e.g. [3]) .

\section{A relationship between the invariants of certain fields}

Let $p>3$ and let $l$ be a prime, $l \equiv 1(\bmod p)$. From now on, we shall assume that $E$ is the abelian field with conductor $l p$, which is of degree $p$ over $P$. Then

Put $t=(l-1) / p$ and denote by $\psi$ a generating character $\bmod l$.

and so

$$
\mathrm{Ch}(E)=\left\{\psi^{t v} \omega^{u} \mid 0 \leqq v \leqq p-1,0 \leqq u \leqq p-2\right\}
$$

$$
X(E)=\left\{\psi^{t v} \omega^{u}|0 \leqq v \leqq p-1,2 \leqq u \leqq p-3,2| u\right\} .
$$

It follows from [11] (Lemma 10) that if $\chi=\psi^{t v} \omega^{u} \in X(E)-X(P)$, then

$$
\begin{aligned}
& S_{n k}(\chi ; E) \equiv \\
& -p^{-n-1} \sum_{i=0}^{s-1}\left[r_{n}\left(i p^{n}+k\right)-r_{n}\left(i p^{n}+k-d_{n}\right)\right] \alpha^{(i+k)(u-1)}(\bmod \mathfrak{p})
\end{aligned}
$$

$\left(n \geqq 0, k \in I_{n}\right)$, where $d_{n}$ is defined by $l \equiv r_{n}\left(d_{n}\right) \quad\left(\bmod p^{n+1}\right)$. This result expresses a certain connection between the $\mu^{-}$-invariants of $E$ and $P$. In fact, we may formulate the following theorem, the first part of which is essentially proved in [11].

Theorem. (i) If $\mu_{\theta}(P)>0$ for some $\theta=\omega^{u} \in X(P)$, then $\mu_{\chi}(E)>0$ for all $\chi=\psi^{t v} \omega^{u} \in X(E)$.

(ii) Suppose that $l \neq \equiv 1\left(\bmod p^{2}\right)$. If $\mu_{x}(E)>0$ for some $\chi=$ $\psi^{t v} \omega^{u} \in X(E)-X(P)$, then $\mu_{\theta}(P)>0$ for $\theta=\omega^{u}$. 
Proof. For the proof of (i), see [11], proof of Theorem 3. We shall now prove (ii). Using Lemma 3 we get that

$$
S_{n k}\left(\psi^{t v} \omega^{u} ; E\right) \equiv 0(\bmod \mathfrak{p})
$$

whenever $n \geqq 0$ and $k \in I_{n}$. This implies, by (9), that

$$
\sum_{i=0}^{s-1} r_{n}\left(i p^{n}+k\right) \alpha^{i(u-1)} \equiv \sum_{i=0}^{s-1} r_{n}\left(i p^{n}+k-d_{n}\right) \alpha^{i(u-1)}\left(\bmod p^{n+2}\right)
$$

for all these $n$ and $k$. In view of (7) and (8) it then follows that

$$
R_{n}(h, u) \equiv R_{n}\left(h-d_{n}, u\right)\left(\bmod p^{n+2}\right)
$$

for all $n \geqq 0$ and all $h \in \mathbb{Z}$.

Since $l \equiv 1 \quad(\bmod p)$, we have $r_{n}\left(d_{n}\right) \equiv 1(\bmod p)$ and so $d_{n}$ is divisible by $p-1 \quad(n \geqq 0)$. On the other hand, $l \not \equiv 1\left(\bmod p^{2}\right)$ so that $d_{n}$ is not divisible by $p$ for $n \geqq 1$. Let us fix some $n \geqq 1$ and some $k \in I_{n}$. Let $z$ satisfy the congruence $d_{n} z \equiv k\left(\bmod p^{n}\right)$. Then

$$
k \equiv k p^{n}+d_{n} z\left(\bmod (p-1) p^{n}\right)
$$

and therefore, by (8),

$$
R_{n}(k, u) \alpha^{k(u-1)}=R_{n}\left(d_{n} z, u\right) .
$$

Combined with (10) this yields

$$
R_{n}(k, u) \alpha^{k(u-1)} \equiv R_{n}(0, u)\left(\bmod p^{n+2}\right) .
$$

We now apply Lemma 4 to rewrite the last congruence as

$$
S_{n k}\left(\omega^{u} ; P\right) \equiv S_{n 0}\left(\omega^{u} ; P\right)(\bmod p) .
$$

Because this holds for all $n \geqq 1$ and $k \in I_{n}$, our assertion follows from the result stated in Remark 4.

Corollary. (i) If $\mu^{-}(P)>0$, then $\mu^{-}(E)>1$.

(ii) Provided that $l \neq 1\left(\bmod p^{2}\right)$, if $\mu^{-}(P)=0$ then $\mu^{-}(E)=0$ and $\lambda^{-}(E) \geqq(p-1)(p-3) / 2$.

Proof. For (i), it is enough to apply part (i) of the theorem to

$$
\mu^{-}(E)=\mu^{-}(P)+e^{-1} \sum_{\chi \in X(E)-X(P)} \mu_{\chi}(E) .
$$

To establish (ii), let $\mu^{-}(P)=0$. Then the result $\mu^{-}(E)=0$ is immediate from part (ii) of the theorem. Moreover, since $d_{0} \equiv 0(\bmod p-1)$ it follows from $(9)$ that $S_{00}(\chi ; E) \equiv 0(\bmod \mathfrak{p})$ for every $\chi \in X(E)-X(P)$. But $\mu_{\chi}(E)=0$ and so the result of Remark 3 tells us that $\lambda_{\chi}(E)>0$. Accordingly, $\lambda-(E)$ is greater than or equal to the number of elements of $X(E)-X(P)$, as was to be proved. 
The above result that $\mu^{-}(P)=0$ implies $\mu^{-}(E)=0$ follows also from a more general result proved by Iwasawa ([9], p. 10) by another method.

As pointed out in [11], it follows from part (i) of the theorem that $\mu^{-}(P)>0$ implies the existence of $\boldsymbol{Z}_{p^{-}}$-extensions $F_{\infty} / F_{0}$ with arbitrarily large $\mu^{-}$. Similarly, if $\mu^{-}(P)=0$, part (ii) gives us $Z_{p}$-extensions $F_{\infty} / F_{0}$ with arbitrarily large $\lambda^{-}$. It also gives, for every regular prime $p$, infinitively many basic $Z_{p}$-extensions $E_{\infty} / E$ with $\mu^{-}=0, \lambda^{-}>0$.

\section{References}

[1] Adachi, N.: Generalization of Kummer's criterion for divisibility of class numbers. - J. Number Theory 5, 1973, 253-265.

[2] Carliti, L.: The first factor of the class number of a cyclic field. - Canad. J. Math. 6, 1954, 23-26.

[3] Gold, R.: The nontriviality of certain $\mathbf{Z}_{l}$-extensions. - J. Number Theory 6, 1974, 369-373.

[4] Hasse, H.: Über die Klassenzahl abelscher Zahlkörper. - Akademie-Verlag, Berlin, 1952.

[5] -》- Vorlesungen über Zahlentheorie. 2. Aufl. - Springer-Verlag, BerlinGöttingen-Heidelberg-New York, 1964.

[6] Iwasawa, K.: On $\Gamma$-extensions of algebraic number fields. - Bull. Amer. Math. Soc. 65, 1959, 183-226.

[7] -»- On $p$-adic $L$-functions. - Ann. of Math. 89, 1969, 198-205.

[8] -》- Lectures on $p$-adic $L$-functions. - Princeton University Press, Princeton, 1972.

[9] - - On the $\mu$-invariants of $\mathbf{Z}_{l}$-extensions. - Number theory, algebraic geometry and commutative algebra, in honor of Y. Akizuki, Kinokuniya, Tokyo, 1973, 1-11.

[10] -»- On $\mathbf{Z}_{l}$-extensions of algebraic number fields. - Ann. of Math. 98, $1973,246-326$.

[11] Metsänky $37,1975,61-75$.

University of Turku

Department of Mathematics

SF-20500 Turku 50

Finland

Received 10 June 1975 